\title{
Effect of miniscrew insertion angle in the maxillary buccal plate on its clinical survival: a randomized clinical trial
}

\author{
Amin Golshah ${ }^{1 *} \mathbb{D}$, Kimia Gorji ${ }^{2}$ and Nafiseh Nikkerdar ${ }^{3}$
}

\begin{abstract}
Introduction: This study sought to assess the effect of miniscrew insertion angle (vertical and oblique) on its clinical survival under shearing forces in orthodontic patients undergoing canine retraction.

Materials and methods: In this split-mouth randomized controlled clinical trial, 50 miniscrews were placed bilaterally in 25 patients with $45^{\circ}$ and $90^{\circ}$ insertion angles relative to a line perpendicular to the occlusal plane distal to the maxillary first premolar extraction site. Allocation of insertion angles to the right/left side was random using the Random Allocation Software. The patients, clinician, and statistician were blinded to the allocation of miniscrews to the side of jaw. The patients were followed-up monthly for 6 months. The primary outcome was the clinical survival of miniscrews, which was evaluated at each follow-up session. The secondary outcomes were the miniscrew stability based on the Periotest value (PTV) and the level of pain experienced by patients at 1, 12, and 24 $\mathrm{h}$, and 7 days after miniscrew placement using a visual analog scale (VAS). Data were analyzed using paired t-test, repeated measures ANOVA, and McNemar's test.
\end{abstract}

Results: The clinical survival rate of miniscrews placed at $90^{\circ}$ and $45^{\circ}$ angles was $76 \%$ and $88 \%$, respectively. This difference was not statistically significant $(P=0.375)$. No significant difference was noted between the two groups regarding the PTV or the pain score either $(P>0.05)$.

Conclusion: Clinically, the insertion angle of miniscrews $\left(90^{\circ}\right.$ versus $45^{\circ}$ relative to a line perpendicular to the occlusal plane) has no significant effect on the miniscrew survival rate or stability during orthodontic treatment.

Trial registration: This trial was registered at www.irct.ir (IRCT20190901044659N1).

Protocol: The protocol was published after trial commencement.

Keywords: Miniscrew, Orthodontic treatment, Clinical survival, Periotest, Visual analog scale

\section{Introduction}

Anchorage in orthodontics simply means prevention of unwanted tooth movements. Miniscrew placement is one suggested strategy to provide orthodontic anchorage [1]. Miniscrew placement is a minimally invasive procedure as long as the risk of contact and traumatization of

\footnotetext{
* Correspondence: amin.golshah@gmail.com

'Department of Orthodontic, School of Dentistry, Kermanshah University of Medical Sciences, Kermanshah, Iran

Full list of author information is available at the end of the article
}

tooth roots is considered. Easy insertion and removal, no dependence on patient cooperation, low cost, and insignificant postoperative pain and discomfort are among the other advantages of miniscrews [1-3]. Due to the aforementioned advantages, miniscrews are extensively used by orthodontists for tooth movement in conventional orthodontic procedures such as molar protraction, canine retraction, correction of dental midline, space closure, and distalization of maxillary molars [1, 4]. However, miniscrews are susceptible to mobility and failure during the treatment course. Several studies have

\section{Springer Open}

(c) The Author(s). 2021 Open Access This article is licensed under a Creative Commons Attribution 4.0 International License, which permits use, sharing, adaptation, distribution and reproduction in any medium or format, as long as you give appropriate credit to the original author(s) and the source, provide a link to the Creative Commons licence, and indicate if changes were made. The images or other third party material in this article are included in the article's Creative Commons licence, unless indicated otherwise in a credit line to the material. If material is not included in the article's Creative Commons licence and your intended use is not permitted by statutory regulation or exceeds the permitted use, you will need to obtain permission directly from the copyright holder. To view a copy of this licence, visit http://creativecommons.org/licenses/by/4.0/. 
evaluated the causes of miniscrew failure; however, the precise factors responsible have not been clearly identified [5-7]. The main factors responsible for miniscrew failure that have been reported so far include inflammation of bone supporting the miniscrew and the adjacent structures, inappropriate site of miniscrew insertion, poor stability due to inadequate cortical bone thickness, bone-related factors, poor oral hygiene status, and age of patients $[8,9]$.

The clinical survival of miniscrews depends on absence of inflammation, detectable mobility or unbearable pain, and the ability of the miniscrew to remain stable and in function under orthodontic forces [7].

The insertion angle is among the suggested factors affecting the clinical survival and primary stability of miniscrews [10-19]. The survival rate of miniscrews has been reported in many previous clinical trials; however, no consensus has been reached on the effect of insertion angle of the miniscrews on their survival rate [7, 20-22].

The available clinical trials on miniscrews have some shortcomings. For instance, the pioneers of this topic did not focus on populations with one single indication for placement or insertion site of miniscrews. Instead, many authors published data derived from all their cases with a mixture of different insertion sites, screws, and indications. However, later trials that focused, for example, on buccal miniscrews for en masse retraction in the maxilla reported higher survival rates [23-25]. Such a variation in survival rates indicates that we should focus on populations with only one indication or treatment concept for miniscrew placement.

\section{Specific objectives}

Considering the role of different factors in clinical survival of miniscrews, the existing controversy in previous studies regarding the effect of miniscrew insertion angle on its clinical survival, and lack of proper clinical trials on this topic, this study aimed to assess the effect of miniscrew insertion angle (vertical and oblique) on its clinical survival rate under shearing forces in orthodontic patients undergoing canine retraction by measuring the miniscrew mobility and pain in the supporting structures. The primary aim was to assess the clinical survival of miniscrews, while the secondary aims were to evaluate the miniscrew stability and the level of pain experienced by patients. The null hypothesis was that the miniscrew insertion angle would have no significant effect on clinical survival or stability, or the level of pain experienced by patients.

\section{Materials and methods}

\section{Trial design}

This was a parallel group, split-mouth, randomized, active-controlled clinical trial with 1:1 allocation ratio.
This study was approved by the ethics committee of Kermanshah University of Medical Sciences (IR.KUMS.REC.1398.694) and registered in the Iranian Registry of Clinical Trials (IRCT20190901044659N1).

\section{Participants, eligibility criteria, and settings}

Twenty-five consecutive patients whose treatment plan included miniscrew insertion in the maxillary buccal plate for maxillary canine retraction into the maxillary first premolar extraction space under shearing orthodontic forces were recruited from a private orthodontic office from December 2019 to August 2020. The inclusion criteria were patients with skeletal class II division 1 malocclusion with molars in a full-cusp class II relationship and normal angle of the maxilla relative to the mandibular plane $\left(25^{\circ} \pm 5^{\circ}\right)$ requiring first premolar extraction and maxillary canine retraction. The exclusion criteria were periodontal disease or bone loss, gross facial asymmetry, cleft lip/palate, impacted or missing teeth at the treatment site, systemic diseases, medication intake, suboptimal frenum position, inadequate length and/or thickness of the attached gingiva, craniofacial disorders of any type, syndromes, systemic diseases, cigarette smoking, and poor oral hygiene. The extractions were performed before the miniscrew placement.

No changes to methods occurred after trial commencement.

\section{Interventions}

After obtaining written informed consent from the patients, the treatment was commenced by using Roth 22 brackets (Discovery, Dentaurum, Germany) and bands (American Orthodontics, USA). After leveling and aligning of the teeth by using the wire sequence of 0.016 nickel titanium, $0.017 \times 0.025$ nickel titanium, and 0.017 $\times 0.025$ stainless steel, the patients were requested to rinse their mouth with $10 \mathrm{cc}$ of $0.2 \%$ chlorhexidine mouthwash (Parodontax, Germany) prior to miniscrew placement. Next, local anesthesia was administered bilaterally by infiltration anesthesia using $2 \%$ lidocaine plus 1:100,000 epinephrine. The miniscrews were then placed at $45^{\circ}$ and $90^{\circ}$ angles relative to a line perpendicular to the occlusal plane into the attached gingiva between the maxillary second premolar and the maxillary first molar (Fig. 1). After bilateral placement of miniscrews, conebeam computed tomography (CBCT) scans were obtained to ensure correct insertion angle of the miniscrews, no contact of miniscrews with the lingual cortical plate or the sinus wall, and adequate distance from the roots (Fig. 2). All CBCT images were obtained with $8 \times$ $8 \mathrm{~cm}$ field of view, 150- $\mu \mathrm{m}$ spatial resolution, $4.19 \mathrm{mAs}$ amperage, and $110 \mathrm{kV}$ voltage. The $\mathrm{CBCT}$ data were exported in DICOM format using the NNT Viewer software version 8 (QR s.r.l, Verona, Italy). A $\pm 5^{\circ}$ error 

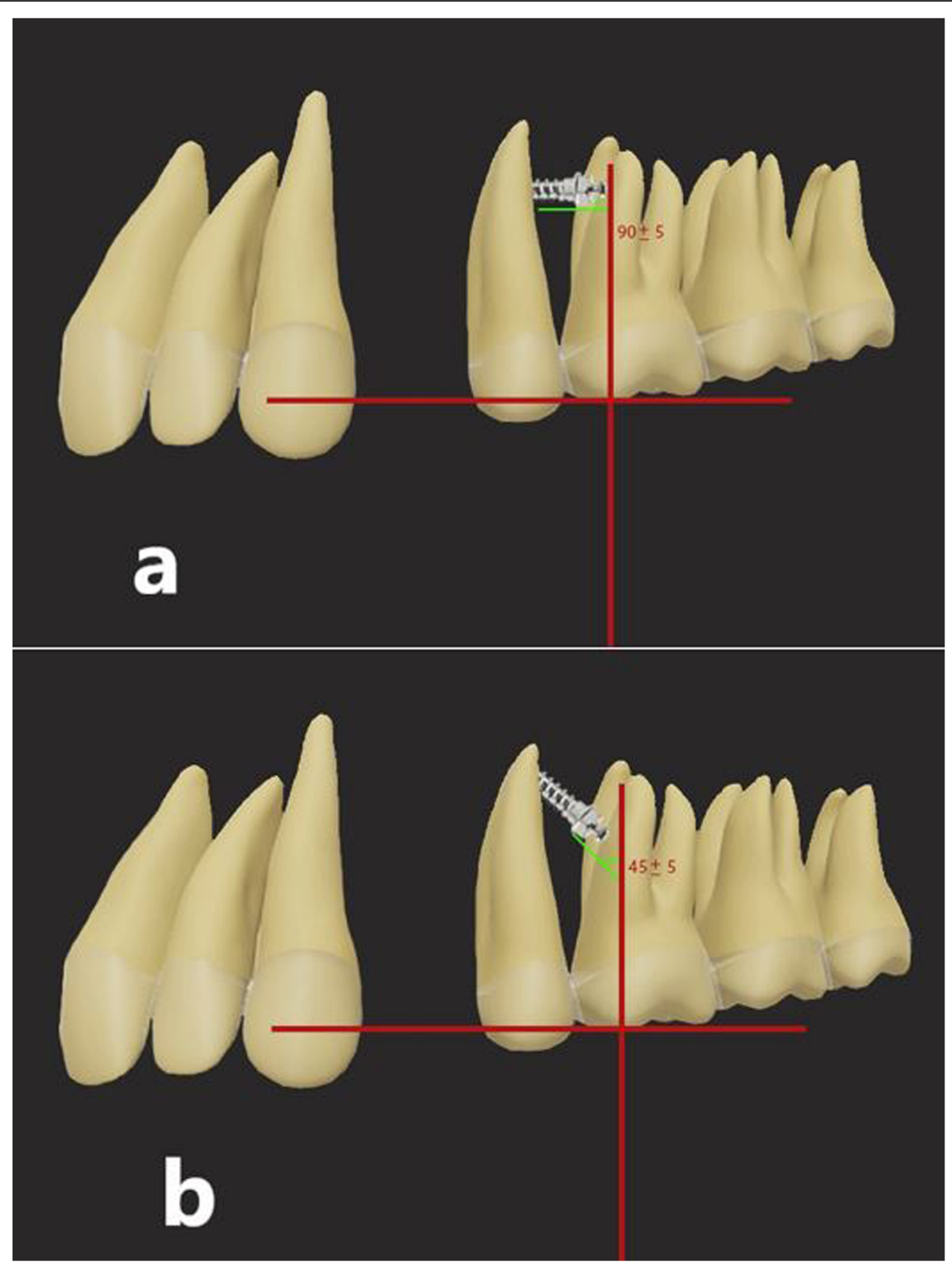

Fig. 1 Schematic view of the insertion of miniscrews: a $90^{\circ}$ insertion angle, b $45^{\circ}$ insertion angle

range was considered acceptable for the insertion angle of miniscrews; otherwise, the case would be replaced. All miniscrews were placed by the same operator (A.G.). The miniscrews used in this study (G2; Jeil, Seoul, South Korea) had $8 \times 1.6 \mathrm{~mm}$ dimensions and were placed in the alveolar bone with an Orthonia driver (Jeil, Seoul, South Korea) with $10 \mathrm{Ncm}$ torque and medium speed (rpm). The patients were instructed to rinse their mouth with $0.12 \%$ chlorhexidine mouthwash twice a day (in the morning and in the evening) for 1 week after the insertion of miniscrews. Also, they were requested to clean the area around the miniscrews after each meal using a soft toothbrush. Two weeks after the placement of miniscrews, canine retraction was started by applying $150 \mathrm{~g}$ force with a nickel-titanium closed coil spring (G\&H orthodontics, Franklin, IN, USA) connecting the miniscrew to the hook of the canine bracket of the corresponding side. The patients were followed up monthly for a total of 6 months. At each follow-up session, after removing the spring, the stability and clinical survival of miniscrews were evaluated by the same operator (K.G.), and the spring was subjected to load application again. At each side, $150 \mathrm{~g}$ force with a nickel-titanium closed coil spring (G\&H orthodontics, Franklin, IN, USA) was applied. At each treatment session, the coil spring was separated from its attachment area to the canine tooth 


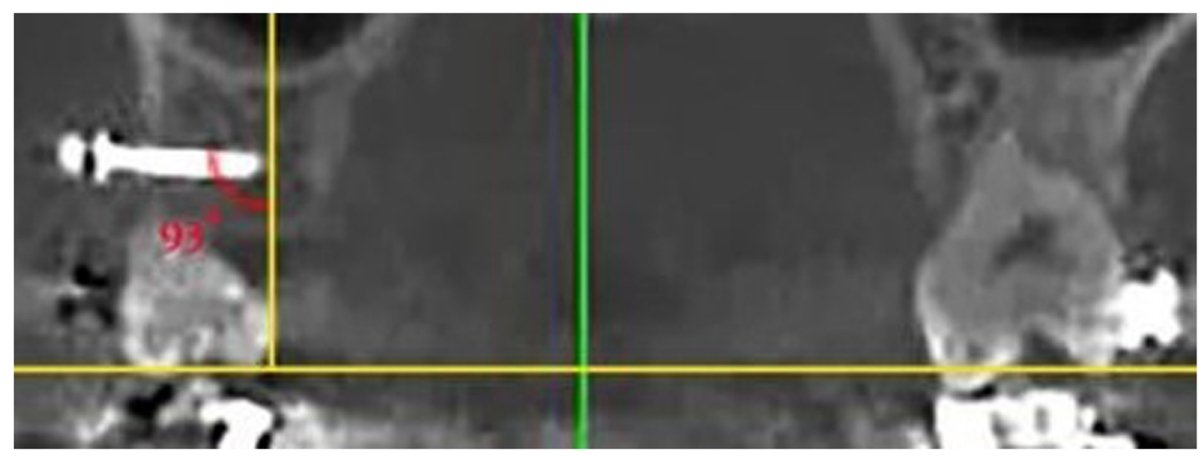

Fig. 2 Coronal cross-sectional CBCT image for measuring the insertion angle of miniscrews

and deactivated. The force was measured by a force meter, and the coil spring was reattached to the canine tooth and activated again.

\section{Outcomes (primary and secondary)}

The primary outcome was to assess the effect of miniscrew insertion angle in the maxillary buccal plate on its clinical survival. The miniscrew clinical survival criteria were as follows:

(I) Absence of signs and symptoms of inflammation such as redness, edema, and exudate

(II) Absence of visible mobility ( $>1 \mathrm{~mm}$ ) indicating that the miniscrew can be used for orthodontic anchorage

(III)Absence of unbearable pain

Presence of any of the abovementioned criteria during the follow-up period was recorded as failure in a checklist. For continuation of patient treatment, the failed miniscrews were removed and reinserted after 1 month, if required.

Assessment of the stability of miniscrews by a Periotest and level of pain experienced by patients using a visual analog scale (VAS) was the secondary outcomes of the study. There were no outcome changes after trial commencement.

\section{Assessment of the level of pain}

The level of pain experienced by patients was measured using a 10-mm VAS at 1,12 , and $24 \mathrm{~h}$, and 7 days after the placement of miniscrews. In case of presence of unbearable pain, it was recorded as failure in the checklist [26].

\section{Assessment of stability}

The stability of miniscrews was evaluated by a Periotest (Medizintechnik Gulden, Germany), which is an electromechanical device for assessment of stability and mobility. The value displayed on the Periotest monitor, referred to as the Periotest value (PTV), can range from
-8 to +50 . The smaller the PTV, the higher the stability; 0 indicates normal physiological mobility while -8 indicates maximum stability [27]. The Periotest was held perpendicular relative to the longitudinal axis of the miniscrew, and three values were recorded at each position (Fig. 3). The mean value was then calculated and recorded as the PTV [27]. The PTV values were measured on a monthly basis during the study period for 6 months.

\section{Sample size calculation}

The minimum sample size was calculated to be $24 \mathrm{pa}$ tients according to a previous study by Park et al. [7] assuming the survival rate of the groups with miniscrew insertion angles of $30-40^{\circ}$ and $90^{\circ}$ to be 0.952 and 0.852 , respectively, alpha $=0.05$, study power of $90 \%$, rate of dropouts to be 0.15 , and accuracy (d) of 0.2 .

\section{Randomization}

Only patients who required bilateral maxillary miniscrews were enrolled in this split-mouth randomized clinical trial. The allocation of miniscrew insertion angles to the left and right maxillary quadrants was random and determined by using the Random Allocation Software (version 2.0; Isfahan, Iran) in 1:1 distribution ratio and equal numbers. Following the random sequence generation, random numbers were allocated to each patient in sealed opaque envelopes. Before the miniscrew placement, the envelope was opened to disclose the angle of miniscrew placement for each maxillary quadrant of the respective patient. The chief of the department, who had no financial, research, or publishing interest in this study, performed the random number generation, allocation, concealment, and implementation.

\section{Blinding}

Allocation of miniscrew angulations to the side of the jaw was random in a triple-blind manner. The patients, the clinician who evaluated the stability and clinical survival of miniscrews (K.G), and the statistician were 


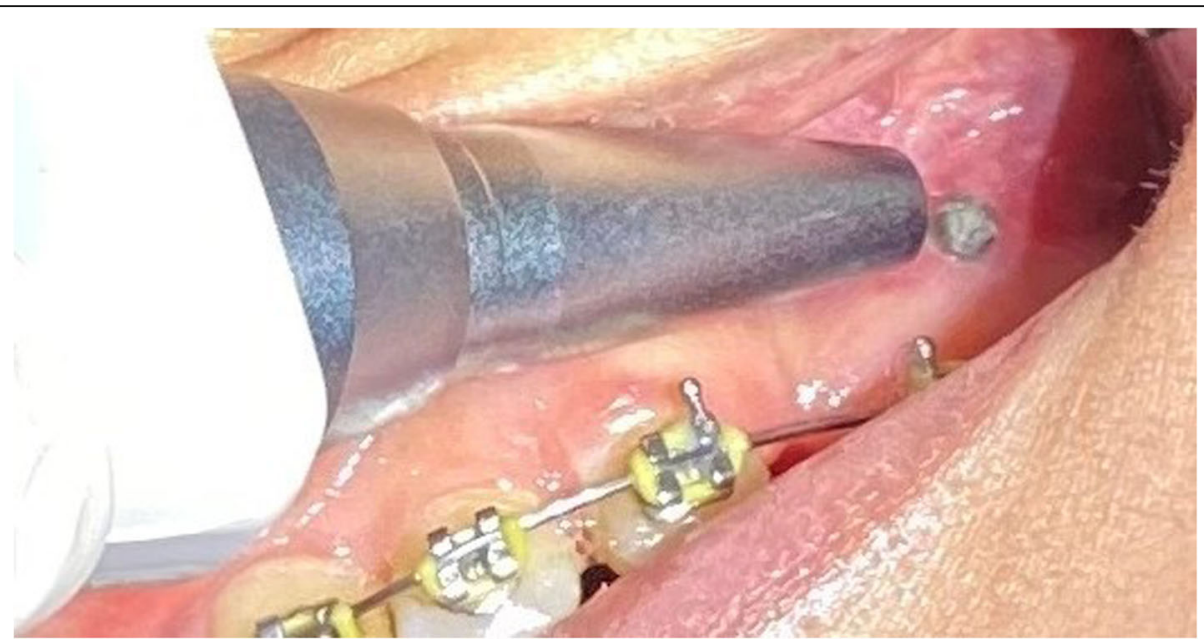

Fig. 3 Periotest was held perpendicular to the longitudinal axis of the miniscrew to evaluate its stability

blinded to the allocation of miniscrews to the side of the jaw.

\section{Statistical analysis}

The Kolmogorov-Smirnov test was applied to assess the normality of data distribution. Since the data were normally distributed, repeated measures ANOVA was used to analyze the changes in PTV over time. The Wilcoxon signed-rank test was applied to compare the pain score between the two quadrants while the Friedman test followed by the Dunn-Bonferroni test was used to analyze the changes in pain score over time. The McNemar test was used to compare the clinical survival of $45^{\circ}$ and $90^{\circ}$ miniscrews as well as the odds ratios at $95 \%$ confidence interval. All statistical analyses were carried out using SPSS version 25 (SPSS Inc., IL, USA) at 0.05 level of significance.

\section{Method error analysis}

For assessment of the reliability of the PTV measurements, $30 \%$ of the cases were remeasured by a highly skilled dental student (K.G.) with the same method. The minimum interclass correlation coefficient was calculated to be 0.933 .

\section{Results}

\section{Participant flow and baseline data}

A total of 36 patients were eligible for participation in this trial. Of these, 11 were excluded since they did not meet the inclusion criteria or were not willing to participate in the study. Thus, this trial enrolled 25 participants including 9 males and 16 females. Figure 4 shows the flow diagram of the study according to the Consolidated Standards of Reporting Trials. One miniscrew was placed in each quadrant of the maxilla (a total of 50).

\section{Primary outcome}

The clinical survival rate of miniscrews placed at $90^{\circ}$ and $45^{\circ}$ angles was $76 \%$ and $88 \%$, respectively, with no significant difference (McNemar test, $P=0.375$ ). Of all, 6 miniscrews (24\%) placed at $90^{\circ}$ angle and 3 miniscrews (12\%) placed at $45^{\circ}$ angle failed.

\section{Secondary outcomes}

Table 1 compares the PTV of $45^{\circ}$ and $90^{\circ}$ miniscrews over time. No significant difference was noted in the PTV of $45^{\circ}$ and $90^{\circ}$ miniscrews at any time point $(P>$ 0.05). Table 2 compares the median pain scores between the 45 and $90^{\circ}$ miniscrew groups at different time points. The median pain score was not significantly different between the two groups at any time point $(P>0.05)$. However, within-group comparisons revealed a significant change in the pain score of patients in the $90^{\circ}$ miniscrew group at different time points $(P<0.001)$, such that the maximum pain score was recorded after $1 \mathrm{~h}$ and then decreased until day 7. Similarly, a significant change was noted in the pain score of patients in the $45^{\circ}$ miniscrew group at different time points $(P<0.001)$ such that the maximum pain score was recorded after $1 \mathrm{~h}$ and then decreased until day 7.

\section{Harms}

No serious harm was observed after miniscrew placement other than pain.

\section{Discussion}

The primary stability of miniscrews is achieved by the mechanical interlocking of their threads in the cortical bone. However, even in case of optimal primary stability, application of excessive loads to the surrounding bone may interfere with the physiological remodeling of the miniscrew surface and bone, and lead to failure [10]. 


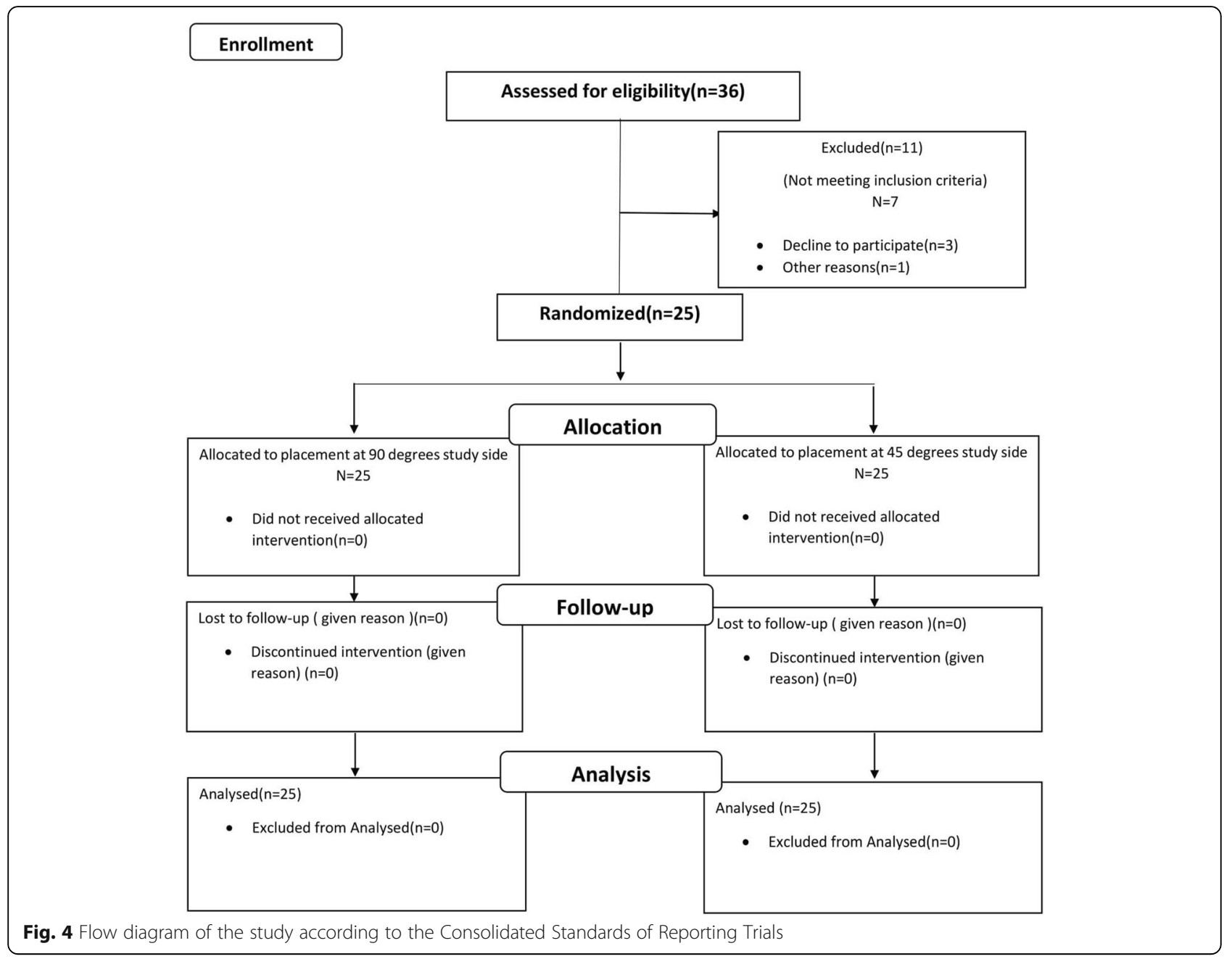

Table 1 Comparison of the PTV of $45^{\circ}$ and $90^{\circ}$ miniscrews over

time

\begin{tabular}{|c|c|c|c|c|c|c|c|}
\hline \multirow[t]{3}{*}{ Time } & \multicolumn{2}{|l|}{$90^{\circ}$} & \multicolumn{2}{|l|}{$45^{\circ}$} & \multirow{2}{*}{\multicolumn{2}{|c|}{$\begin{array}{l}95 \% \text { confidence } \\
\text { interval }\end{array}$}} & \multirow[t]{3}{*}{$P$-value ${ }^{\dagger}$} \\
\hline & \multirow{2}{*}{ Mean } & \multirow[t]{2}{*}{ SD } & \multirow{2}{*}{ Mean } & \multirow{2}{*}{ SD } & & & \\
\hline & & & & & Lower & Upper & \\
\hline At 1 month & $4.07^{a}$ & 7.76 & $3.72^{a}$ & 9.19 & -4.28 & 4.71 & 0.923 \\
\hline At 2 months & $4.64^{\mathrm{a}}$ & 7.94 & $4.64^{\mathrm{a}}$ & 9.76 & -4.82 & 4.42 & 0.929 \\
\hline At 3 months & $5.66^{\mathrm{a}}$ & 6.96 & $4.04^{\mathrm{a}}$ & 7.85 & -2.63 & 5.56 & 0.466 \\
\hline At 4 months & $5.20^{a}$ & 6.25 & $4.07^{a}$ & 5.89 & -1.29 & 2.90 & 0.432 \\
\hline At 5 months & $4.71^{\mathrm{a}}$ & 6.83 & $3.68^{a}$ & 5.66 & -2.67 & 4.13 & 0.656 \\
\hline At 6 months & $2.95^{\mathrm{a}}$ & 5.06 & $6.01^{a}$ & 5.13 & -8.12 & 1.40 & 0.150 \\
\hline$P$-value ${ }^{\neq}$ & 0.104 & & 0.160 & & & & \\
\hline
\end{tabular}

\section{${ }^{\dagger}$ Paired t-test}

${ }^{\ddagger}$ Repeated measures ANOVA followed by LSD test

Means with the same superscript letters are not significantly different $(P$ $>0.05$ )
The failure rate of miniscrews is variable in the literature. For example, Papageorgiou et al. [28] reported a failure rate of $13.5 \%$ while this rate was $14.5 \%$ in a study by Motoyoshi et al. [29] and $14.2 \%$ in a study by Watanabe et al. [22].

Table 2 Comparison of the median pain scores between the 45 and $90^{\circ}$ miniscrew groups at different time points

\begin{tabular}{|c|c|c|c|c|c|}
\hline \multirow[t]{2}{*}{ Time } & \multicolumn{2}{|l|}{$90^{\circ}$} & \multicolumn{2}{|l|}{$45^{\circ}$} & \multirow[t]{2}{*}{$P$-value ${ }^{\dagger}$} \\
\hline & Median & IQR & Median & IQR & \\
\hline At $1 \mathrm{~h}$ & $4^{c}$ & 1 & $4^{c}$ & 2 & 0.511 \\
\hline At $12 \mathrm{~h}$ & $2^{b}$ & 1 & $2^{b}$ & 1 & 0.595 \\
\hline At $24 \mathrm{~h}$ & $1^{\mathrm{ab}}$ & 2 & $1^{a b}$ & 1 & 0.108 \\
\hline At 7 days & $0^{a}$ & 0 & $0^{a}$ & 0 & 0.317 \\
\hline$P$-value ${ }^{\neq}$ & $<0.001$ & & $<0.001$ & & \\
\hline
\end{tabular}

${ }^{\dagger}$ Wilcoxon signed-rank test

${ }^{\ddagger}$ Friedman test followed by Dunn's multiple comparisons test (significant values have been adjusted by the Bonferroni correction for multiple tests) Medians with the same superscript letters are not significantly different $(P$ $>0.05$ )

IQR interquartile range 
The survival rate of miniscrews reported in the literature is often over $80 \%$ [30-34]. The variable range of clinical survival rate of miniscrews may be due to different survival and failure criteria in different studies and variability in study populations in terms of age, gender, anatomical position of miniscrew, type of malocclusion, and oral hygiene status [35]. Also, miniscrew-related factors such as type, length, and diameter of miniscrews are variable [36]. Moreover, the magnitude and duration of load application and type of orthodontic movement also vary in different studies [37].

This study assessed the effect of miniscrew insertion angle (vertical and oblique) on its clinical survival under shearing forces in orthodontic patients. Clinically, the insertion angle of miniscrews $\left(90^{\circ}\right.$ versus $\left.45^{\circ}\right)$ had no significant effect on the miniscrew survival rate or stability during orthodontic treatment, or the level of pain experienced by patients. Thus, the null hypothesis of the study was accepted. Optimal stability is one of the survival criteria of miniscrews [10]. Based on this fact, some in vitro studies investigated the effect of insertion angle of miniscrews on their stability and reported controversial results. Some studies showed that oblique insertion of miniscrews decreased their stability [14-16, 38-40], while some others reported that oblique placement of miniscrews increased their stability [10-13, 41-44]. The results of these studies were different from our findings, which may be due to their in vitro design and poor generalizability of their results to the clinical setting. Recently, an in vitro study evaluated the effect of size, technique of placement, and insertion angle of miniscrews on primary stability of mini-implants. They discussed that the primary stability of miniscrews depended on the density of the cancerous bone. They found that angulation of miniscrew in high-density bone decreased the primary stability, but this was not the case for the low-density bone. Thus, they recommended assessment of cancellous bone density prior to miniscrew placement [45].

In line with our results, some previous clinical trials demonstrated that the insertion angle had no significant effect on miniscrew survival [7, 20-22]. The main shortcoming of such trials was that they did not focus on populations with uniform insertion sites, screws, or indications, whereas the present trial only focused on skeletal class II division 1 malocclusion patients with molars in a full-cusp class II relationship and normal angle of the maxilla relative to the mandibular plane $\left(25^{\circ} \pm 5^{\circ}\right)$. The patients required first premolar extraction and maxillary canine retraction by using bilateral maxillary miniscrews placed in the maxillary buccal plate. The manufacturing company and dimensions of all inserted miniscrews were the same (G2; Jeil, Seoul, South Korea, $8 \times 1.6 \mathrm{~mm}$ ). Also, the miniscrews were loaded by equal shearing orthodontic forces in all patients (150 g). By doing so, the confounding effect of all these variables on the results was eliminated.

In this study, Periotest was used to assess the stability of miniscrews, which showed that the miniscrew insertion angle had no significant effect on the PTV. Optimal clinical application of Periotest for assessment of miniscrew stability was first confirmed in an in vitro study [46]. The authors reported that the insertion torque was correlated with the PTV, such that the higher the insertion torque in bone, the lower the PTV and the higher the primary stability would be [46]. Watanabe et al. [47] confirmed this finding and suggested the PTV, the amount of insertion torque, and the cortical bone thickness as the criteria for clinical stability and survival prediction of miniscrews. According to Atsumi et al. [48], Periotest is a non-invasive quantitative tool with maximum efficacy for measurement of the primary stability of dental implants.

Measurement of pain by VAS in this study revealed that the pain score was maximum at $1 \mathrm{~h}$ after miniscrew placement and decreased over time in both groups. Mirhashemi et al. [49] in their clinical study evaluated the pain score following miniscrew insertion in orthodontic patients and reported maximum pain at $1 \mathrm{~h}$ after miniscrew placement; the pain score had a descending trend after that for 7 days. Kuroda et al. [1] reported maximum pain at $1 \mathrm{~h}$ after miniscrew placement and its decreasing trend for up to 7 days. The abovementioned results support our findings. Łyczek et al. [50] evaluated the level of pain and inflammation following miniscrew insertion with $45^{\circ}$ angle and reported mild pain and discomfort after 1 day.

In total, since none of the tested insertion angles of miniscrews had any superiority over the other, clinicians can place the miniscrews vertically or obliquely based on the clinical and anatomical patient conditions, position of the teeth and roots, and type of applied force, and this decision would not affect the clinical survival of miniscrews.

Future clinical trials with larger sample size are required to assess the effect of other types of orthodontic forces in different miniscrew insertion angles on their clinical survival rate. Moreover, a recent study reported computer-guided placement of mini-implants [51], which calls for further studies regarding the clinical survival and possible complications of miniscrews placed by this technique.

\section{Limitations}

Small sample size and not measuring the level of proinflammatory cytokines in the gingival crevicular fluid were among the limitations of this study. Future studies with larger sample size and multi-center design are required to measure the level of pro-inflammatory 
cytokines in the gingival crevicular fluid following miniscrew placement at different angles. Also, the miniscrews were loaded 2 weeks after placement in this study, which may not be an ideal time since at this moment primary stability probably decreases while secondary stability is not yet present. Future studies are required to assess the mini-screw stability immediately after loading [52].

\section{Generalizability}

The generalizability of the current results might be limited because this study was undertaken in one center by one clinician (A.G.) experienced in placing miniscrews.

\section{Conclusion}

According to the results, the clinical survival rate and the stability of miniscrews and the level of postoperative pain and discomfort experienced by patients were not significantly different when oblique $\left(45^{\circ}\right)$ and vertical $\left(90^{\circ}\right)$ insertion angles were compared.

\section{Abbreviations}

PTV: Periotest value; VAS: Visual analog scale; CBCT: Cone-beam computed tomography

\section{Acknowledgements}

This study was supported by the Kermanshah University of Medical Science, Kermanshah, Iran.

\section{Authors' contributions}

AG devised the study concept; designed the study; acquired data; drafted the manuscript; critically revised the manuscript for important intellectual content; and contributed to administrative, technical, and material support and study supervision. KG devised analysis and interpretation of data, drafted the manuscript, and contributed to statistical analysis and administrative, technical, and material support. NN devised the study concept and design, drafted the manuscript, critically revised the manuscript for important intellectual content, and contributed to administrative, technical, and material support. The authors read and approved the final manuscript.

\section{Funding}

This trial received funding from Kermanshah University of Medical Sciences.

\section{Availability of data and materials}

The datasets generated and/or analyzed during the current study are not publicly available because of the privacy of patients but are available from the corresponding author on reasonable request.

\section{Declarations}

Ethics approval and consent to participate

The study protocol was approved by the ethics committee of Kermanshah University of Medical Sciences (IR.KUMS.REC.1398.694).

\section{Consent for publication}

All authors consent to publication of this manuscript.

\section{Competing interests}

The authors declare that they have no competing interests.

\section{Author details}

'Department of Orthodontic, School of Dentistry, Kermanshah University of Medical Sciences, Kermanshah, Iran. ${ }^{2}$ Students Research Committee, School of Dentistry, Kermanshah University of Medical Sciences, Kermanshah, Iran. ${ }^{3}$ Department of Oral and Maxillofacial Radiology, School of Dentistry, Kermanshah University of Medical Sciences, Kermanshah, Iran.
Received: 12 April 2021 Accepted: 18 June 2021

Published online: 02 August 2021

\section{References}

1. Kuroda S, Sugawara Y, Deguchi T, Kyung HM, Takano-Yamamoto T. Clinical use of miniscrew implants as orthodontic anchorage: success rates and postoperative discomfort. Am J Orthod Dentofacial Orthop. 2007;131(1):915. https://doi.org/10.1016/j.ajodo.2005.02.032.

2. Elias CN, de Oliveira Ruellas AC, Fernandes DJ. Orthodontic implants: concepts for the orthodontic practitioner. Int J Dent. 2012;2012:549761.

3. Yamaguchi $M$, Inami $T$, Ito K, Kasai K, Tanimoto $Y$. Mini-implants in the anchorage armamentarium: new paradigms in the orthodontics. Int J Biomater. 2012:2012:394121.

4. Kılınç DD, Sayar G. Various contemporary intraoral anchorage mechanics supported with temporary anchorage devices. Turk J Orthod. 2016;29(4): 109-13. https://doi.org/10.5152/TurkJOrthod.2016.16027.

5. Lim HJ, Eun $\mathrm{CS}$, Cho JH, Lee KH, Hwang HS. Factors associated with initial stability of miniscrews for orthodontic treatment. Am J Orthod Dentofacia Orthop. 2009;136(2):236-42. https://doi.org/10.1016/j.ajodo.2007.07.030.

6. Topouzelis N, Tsaousoglou P. Clinical factors correlated with the success rate of miniscrews in orthodontic treatment. Int J Oral Sci. 2012;4(1):38-44. https://doi.org/10.1038/ijos.2012.1.

7. Park HS, Jeong SH, Kwon OW. Factors affecting the clinical success of screw implants used as orthodontic anchorage. Am J Orthod Dentofacial Orthop. 2006;130(1):18-25. https://doi.org/10.1016/j.ajodo.2004.11.032.

8. Sung JH, Kyung HM, Bae SM, Park HS, Kwon OW, McNamara JA. Clinical examples of microimplant anchorage. Microimplants in orthodontics. Daegu: Dentos; 2006. p. 83-163.

9. Papadopoulos MA. Orthodontic treatment of class II malocclusion with miniscrew implants. Am J Orthod Dentofacial Orthop. 2008;134:604.e1-16 discussion 604-5.

10. Wilmes B, Neuschulz J, Safar M, Braumann B, Drescher D. Protocols for combining the Beneslider with lingual appliances in class II treatment. J Clin Orthod. 2014;48(12):744-52.

11. Lim JE, Lim WH, Chun YS. Quantitative evaluation of cortical bone thickness and root proximity at maxillary interradicular sites for orthodontic miniimplant placement. Clin Anat. 2008;21(6):486-91. https://doi.org/10.1002/ ca.20671.

12. Liou EJ, Chen PH, Wang YC, Lin JC. A computed tomographic image study on the thickness of the infrazygomatic crest of the maxilla and its clinical implications for miniscrew insertion. Am J Orthod Dentofacial Orthop. 2007; 131(3):352-6. https://doi.org/10.1016/j.ajodo.2005.04.044.

13. Deguchi T, Nasu M, Murakami K, Yabuuchi T, Kamioka H, Takano-Yamamoto T. Quantitative evaluation of cortical bone thickness with computed tomographic scanning for orthodontic implants. Am J Orthod Dentofacial Orthop. 2006:129:721.e7-12.

14. Jasmine MI, Yezdani AA, Tajir F, Venu RM. Analysis of stress in bone and microimplants during en-masse retraction of maxillary and mandibular anterior teeth with different insertion angulations: a 3-dimensional finite element analysis study. Am J Orthod Dentofacial Orthop. 2012;141(1):71-80. https://doi.org/10.1016/j.ajodo.2011.06.031

15. Pickard MB, Dechow P, Rossouw PE, Buschang PH. Effects of miniscrew orientation on implant stability and resistance to failure. Am J Orthod Dentofacial Orthop. 2010;137(1):91-9. https://doi.org/10.1016/j.ajodo.2007.12. 034.

16. Lee J, Kim JY, Choi YJ, Kim KH, Chung CJ. Effects of placement angle and direction of orthopedic force application on the stability of orthodontic miniscrews. Angle Orthod. 2013;83(4):667-73. https://doi.org/10.2319/ 090112-703.1.

17. Shinohara A, Motoyoshi M, Uchida Y, Shimizu N. Root proximity and inclination of orthodontic mini-implants after placement: cone-beam computed tomography evaluation. Am J Orthod Dentofacial Orthop. 2013; 144(1):50-6. https://doi.org/10.1016/j.ajodo.2013.02.021.

18. Kim SH, Kang SM, Choi YS, Kook YA, Chung KR, Huang JC. Cone-beam computed tomography evaluation of mini-implants after placement: is root proximity a major risk factor for failure? Am J Orthod Dentofacial Orthop. 2010;138(3):264-76. https://doi.org/10.1016/j.ajodo.2008.07.026.

19. Jung YR, Kim SC, Kang KH, Cho JH, Lee EH, Chang NY, et al. Placement angle effects on the success rate of orthodontic microimplants and other factors with conebeam computed tomography. Am J Orthod Dentofacial Orthop. 2013;143(2):173-81. https://doi.org/10.1016/j.ajodo.2012.09.011. 
20. Jing $Z$, Wu $Y$, Jiang $W$, Zhao $L$, Jing $D$, Zhang $N$, et al. Factors affecting the clinical success rate of miniscrew implants for orthodontic treatment. Int J Oral Maxillofac Implants. 2016;31(4):835-41. https://doi.org/10.11607/jomi.4197.

21. Miyawaki S, Koyama I, Inoue M, Mishima K, Sugahara T, Takano-Yamamoto T. Factors associated with the stability of titanium screws placed in the posterior region for orthodontic anchorage. Am J Orthod Dentofacial Orthop. 2003;124(4):373-8. https://doi.org/10.1016/s0889-5406(03)00565-1.

22. Watanabe H, Deguchi T, Hasegawa M, Ito M, Kim S, Takano-Yamamoto T. Orthodontic miniscrew failure rate and root proximity, insertion angle, bone contact length, and bone density. Orthod Craniofac Res. 2013;16(1):44-55. https://doi.org/10.1111/ocr.12003.

23. Al-Sibaie S, Hajeer MY. Assessment of changes following en-masse retraction with mini-implants anchorage compared to two-step retraction with conventional anchorage in patients with class II division 1 malocclusion: a randomized controlled trial. Eur J Orthod. 2014;36(3):275-83. https://doi.org/10.1093/ejo/cjt046.

24. Basha AG, Shantaraj R, Mogegowda SB. Comparative study between conventional en-masse retraction (sliding mechanics) and en-masse retraction using orthodontic micro implant. Implant Dent. 2010;19(2):12836. https://doi.org/10.1097/ID.0b013e3181cc4aa5.

25. Chopra SS, Mukherjee M, Mitra R, Kochar GD, Kadu A. Comparative evaluation of anchorage reinforcement between orthodontic implants and conventional anchorage in orthodontic management of bimaxillary dentoalveolar protrusion. Med J Armed Forces India. 2017;73(2):159-66. https://doi.org/10.1016/j.mjafi.2016.01.003.

26. Attri S, Mittal R, Batra P, Sonar S, Sharma K, Raghavan S, et al. Comparison of rate of tooth movement and pain perception during accelerated tooth movement associated with conventional fixed appliances with microosteoperforations - a randomised controlled trial. J Orthod. 2018;45(4):22533. https://doi.org/10.1080/14653125.2018.1528746.

27. Swami V, Vijayaraghavan V, Swami V. Current trends to measure implant stability. J Indian Prosthodont Soc. 2016;16(2):124-30. https://doi.org/10.41 03/0972-4052.176539.

28. Papageorgiou SN, Zogakis IP, Papadopoulos MA. Failure rates and associated risk factors of orthodontic miniscrew implants: a meta-analysis. Am J Orthod Dentofacial Orthop. 2012;142:577-95.e7.

29. Motoyoshi M, Hirabayashi M, Uemura M, Shimizu N. Recommended placement torque when tightening an orthodontic mini-implant. Clin Oral Implants Res. 2006;17(1):109-14. https://doi.org/10.1111/j.1600-0501.2005. 01211.x.

30. Moon CH, Lee DG, Lee HS, Im JS, Baek SH. Factors associated with the success rate of orthodontic miniscrews placed in the upper and lower posterior buccal region. Angle Orthod. 2008;78(1):101-6. https://doi.org/1 0.2319/121706-515.1

31. Luzi C, Verna C, Melsen B. A prospective clinical investigation of the failure rate of immediately loaded mini-implants used for orthodontic anchorage. Prog Orthod. 2007:8(1):192-201.

32. Hedayati Z, Hashemi SM, Zamiri B, Fattahi HR. Anchorage value of surgical titanium screws in orthodontic tooth movement. Int J Oral Maxillofac Surg. 2007;36(7):588-92. https://doi.org/10.1016/j.jom.2006.10.020.

33. Wiechmann $D$, Meyer $U$, Büchter A. Success rate of mini- and microimplants used for orthodontic anchorage: a prospective clinical study. Clin Oral Implants Res. 2007;18(2):263-7. https://doi.org/10.1111/j.1600-0501.2006 01325.x.

34. Chen $\mathrm{CH}$, Chang $\mathrm{CS}$, Hsieh $\mathrm{CH}$, Tseng YC, Shen YS, Huang IY, et al. The use of microimplants in orthodontic anchorage. J Oral Maxillofac Surg. 2006; 64(8):1209-13. https://doi.org/10.1016/j.joms.2006.04.016.

35. Chen Y, Kyung HM, Zhao WT, Yu WJ. Critical factors for the success of orthodontic mini-implants: a systematic review. Am J Orthod Dentofacial Orthop. 2009;135(3):284-91. https://doi.org/10.1016/j.ajodo.2007.08.017

36. Reynders R, Ronchi L, Bipat S. Mini-implants in orthodontics: a systematic review of the literature. Am J Orthod Dentofacial Orthop. 2009;135:564.e119 discussion 564-5.

37. Schätzle M, Männchen R, Zwahlen M, Lang NP. Survival and failure rates of orthodontic temporary anchorage devices: a systematic review. Clin Oral Implants Res. 2009;20(12):1351-9. https://doi.org/10.1111/j.1600-0501.2009.01 754.x

38. Petrey JS, Saunders MM, Kluemper GT, Cunningham LL, Beeman CS. Temporary anchorage device insertion variables: effects on retention. Angle Orthod. 2010;80(4):446-53. https://doi.org/10.2319/070309-376.1.
39. Noble JA, Karaiskos NE, Hassard TH, Hechter FJ, Wiltshire WA. Stress on bone from placement and removal of orthodontic miniscrews at different angulations. J Clin Orthod. 2009;43(5):332-4

40. Bayerly I, Khalil F. Effect of dual-thread, length and insertion angle in orthodontic miniscrews primary stability-an in vitro study. Int Arab J Dent. 2015;6(1). https://ojs.usj.edu.lb/ojs/index.php/iajd/article/view/208. Accessed 02 Jul 2021.

41. Araghbidikashani M, Golshah A, Nikkerdar N, Rezaei M. In-vitro impact of insertion angle on primary stability of miniscrews. Am J Orthod Dentofacial Orthop. 2016;150(3):436-43. https://doi.org/10.1016/j.ajodo.2016.02.020.

42. Zhao L, Xu Z, Wei X, Zhao Z, Yang Z, Zhang L, et al. Effect of placement angle on the stability of loaded titanium microscrews: a microcomputed tomographic and biomechanical analysis. Am J Orthod Dentofacial Orthop. 2011;139(5):628-35. https://doi.org/10.1016/j.ajodo.2009.06.040.

43. Tatli U, Alraawi M, Toroğlu MS. Effects of size and insertion angle of orthodontic mini-implants on skeletal anchorage. Am J Orthod Dentofacial Orthop. 2019;156(2):220-8. https://doi.org/10.1016/j.ajodo.2018.08.026.

44. Raji SH, Noorollahian S, Niknam SM. The effect of insertion angle on orthodontic mini-screw torque. Dent Res J (Isfahan). 2014;11(4):448-51.

45. Möhlhenrich SC, Heussen N, Modabber A, Bock A, Hölzle F, Wilmes B, et al. Influence of bone density, screw size and surgical procedure on orthodontic mini-implant placement - part B: implant stability. Int J Oral Maxillofac Surg. 2021;50(4):565-72. https://doi.org/10.1016/j.ijom.2020.07.003.

46. Çehreli S, Arman-Özçırpıcı A. Primary stability and histomorphometric boneimplant contact of self-drilling and self-tapping orthodontic microimplants. Am J Orthod Dentofacial Orthop. 2012;141(2):187-95. https://doi.org/10.101 6/j.ajodo.2011.07.020.

47. Watanabe T, Miyazawa K, Fujiwara T, Kawaguchi M, Tabuchi M, Goto S. Insertion torque and Periotest values are important factors predicting outcome after orthodontic miniscrew placement. Am J Orthod Dentofacial Orthop. 2017;152(4):483-8. https://doi.org/10.1016/j.ajodo.2017.01.026.

48. Atsumi M, Park SH, Wang HL. Methods used to assess implant stability: current status. Int J Oral Maxillofac Implants. 2007:22(5):743-54.

49. Mirhashemi A, Hosseini MH, Yadalloahi H, Jalali YF. Pain and discomfort experience after miniscrew insertion as an anchorage device in orthodontic patients. Dental Hypotheses. 2016;7:112.

50. Łyczek J, Kawala B, Antoszewska-Smith J. Influence of antibiotic prophylaxis on the stability of orthodontic microimplants: a pilot randomized controlled trial. Am J Orthod Dentofacial Orthop. 2018;153(5):621-31. https://doi.org/1 0.1016/j.ajodo.2017.11.025

51. Altieri F, Padalino G, Guarnieri R, Barbato E, Cassetta M. Computer-guided palatal canine disimpaction: a technical note. Int J Comput Dent. 2020;23(3): 219-24.

52. Cassetta M, Brandetti G, Altieri F. Is a two-month healing period long enough to achieve osseointegration? A prospective clinical cohort study. Int J Oral Maxillofac Surg. 2020;49(5):649-54. https://doi.org/10.1016/j.ijom.201 9.10.020.

\section{Publisher's Note}

Springer Nature remains neutral with regard to jurisdictional claims in published maps and institutional affiliations.

\section{Submit your manuscript to a SpringerOpen ${ }^{\circ}$ journal and benefit from:}

- Convenient online submission

- Rigorous peer review

- Open access: articles freely available online

High visibility within the field

- Retaining the copyright to your article

Submit your next manuscript at $>$ springeropen.com 